\title{
Tricks and Treats: Designing Technology to Support Mobility Assistance Dogs
}

\author{
Charlotte Robinson \\ charlotte.robinson@sussex.ac.uk \\ University of Sussex \\ Falmer, United Kingdom
}

\author{
Alice Torjussen \\ at459@sussex.ac.uk \\ University of Sussex \\ Falmer, United Kingdom
}

\author{
Emeline Brulé \\ e.t.brule@sussex.ac.uk \\ University of Sussex \\ Falmer, United Kingdom
}

\author{
Joshua Kybett \\ jtk22@sussex.ac.uk \\ University of Sussex \\ Falmer, United Kingdom
}

\author{
James Jackson \\ jaj30@sussex.ac.uk \\ University of Sussex \\ Falmer, United Kingdom
}

\author{
Tom Appshaw \\ ta308@sussex.ac.uk \\ University of Sussex \\ Falmer, United Kingdom
}
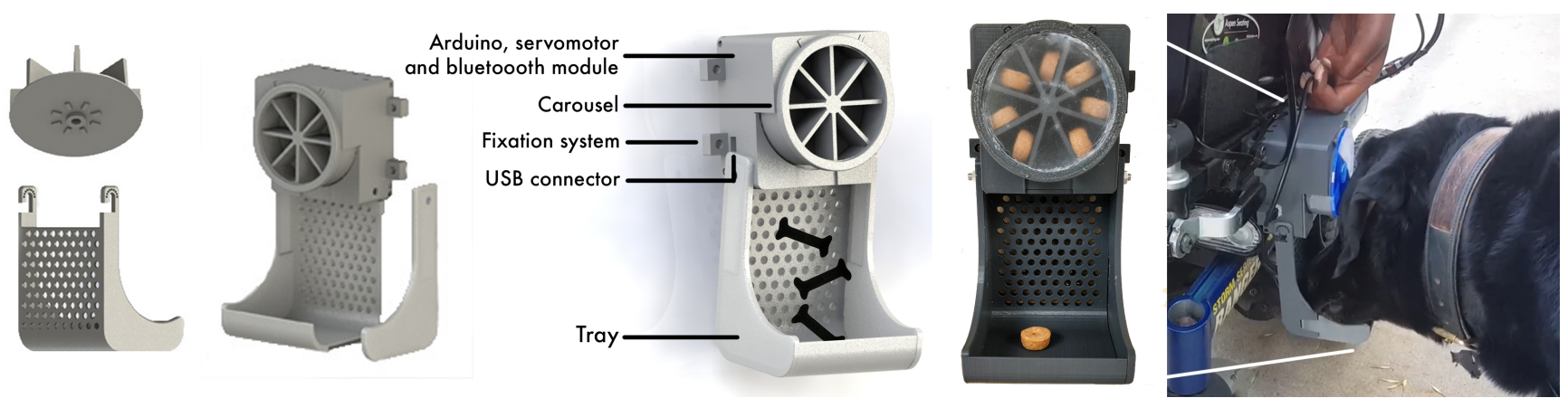

Figure 1. From left: 3D model showing the underside of the carousel with the raised notches, 3D model of the previous tray design which used hooks to attach to the main module, 3D model of the final tray design which attaches using a nut/bolt mechanism, 3D model of the final treat dispenser, and a photo of the 3D printed final dispenser deployed to participants.

\begin{abstract}
Assistance dogs are a key intervention to support the autonomy of people with tetraplegia. Previous research on assistive technologies have investigated ways to, ultimately, replace their labour using technology, for instance through the design of smart home environments. However, both the disability studies literature and our interviews suggest there is an immediate need to support these relationships, both in terms of training and bonding. Through a case study of an accessible dog treats dispenser, we investigate a technological intervention responding to these needs, detailing an appropriate design methodology and contributing insights into user requirements and preferences.
\end{abstract}

\section{Author Keywords}

Assistance Dog; Service Dog; Tetraplegia; Assistive Technology; Disability

\section{CCS Concepts}

-Human-centered computing $\rightarrow$ Accessibility systems and tools; $\cdot$ Social and professional topics $\rightarrow$ People with disabilities;

\section{INTRODUCTION}

Assistance dogs are matched to people with disabilities to support autonomy in daily life. These dogs can accomplish many tasks, such as helping their owners navigate physical environments or responding to medical emergencies [82, 59, 66]. Work in HCI and assistive technology has sought to leverage technology to accomplish these same tasks, often through the design of smart-home environments (e.g. [72, 12]) or robotics (e.g. [51, 52]). However, disability research suggests the benefits of assistance dogs go well beyond the practical tasks they accomplish[61, 83, 17, 58, 79], providing companionship and flexibility beyond anything that can currently be achieved by technological systems. This work thus investigated challenges that people with disabilities and their dogs currently face as working partnerships, taking into consideration any current practice and use of assistive technologies.

While assistance dogs support a wide range of impairments (visual and/or motor impairments [43, 82], diabetes [60, 62], seizure disorders [15], autism [48], etc.), this work identified 
a specific need to support people with tetraplegia, who use wheelchairs and have limited use of upper extremities. For these individuals, there may be heightened challenges physically interacting with their dog (i.e. petting or giving food rewards). This, in turn, may create additional challenges in training and bonding.

We hence investigated potential technological interventions to facilitate bonding between people with tetraplegia and their dogs. Following this initial investigation of the design space, we propose a new treat dispenser system that would address these additional challenges of training and bonding in such partnerships. The system was designed and evaluated via a participatory design process alongside people with tetraplegia and their dogs. We modified our participatory design process for the unique needs of the participants and the presence of multi-species (canine and human) users, thus providing further methodological insights about designing systems that support both canine and human users. Preliminary data showed a positive response to users and their dogs' ability to use the system we developed. Using feedback from this initial prototype, we further iterated on the system, and deployed devices with participants to further understand the potential impact such a device could have on on these human-canine partnerships. We found that such an intervention had the capacity to positively impact the lives of individuals with tetraplegia and that there are additional potential opportunities to help similar user groups. Through this work, we aim to further integrate the fields of assistive technology and Canine-Computer Interaction, that have intersecting goals of supporting humans in their daily living tasks, and supporting humans in their relationships with dogs.

\section{BACKGROUND}

Here, we introduce the context of this study by describing assistance dog training frameworks, and by providing information about people with tetraplegia and their current practices with assistance dogs. We then discuss work that has developed accessible technologies for people with tetraplegia, identifying a gap in the research work: namely the investigation of potential technologies to support human bonding and collaboration with assistance dogs for this particular application. We also consider existing design and methodological guidelines for canine-computer interaction, which inform our work.

\section{Context}

People with tetraplegia

The World Health Organisation (WHO) estimates that globally each year, between 250,000 and 500,000 people suffer a spinal cord injury (SCI) [49]. Tetraplegia occurs after an upper spinal cord injury or illness causes partial or total paralysis in all four limbs and torso [70]. The majority of research into the quality of life of individuals with tetraplegia or following a SCI adopts a qualitative approach, relying on methods such as interviews and questionnaires for data collection[27]. This is because quality of life is often considered a subjective construct, defined by one's perception of factors such as physical well-being and independence, emotional well-being, and relationships and social function [27, 44]. For example, energy expenditure for completing everyday tasks plays an important role in personal independence, which significantly impacts quality of life. Even when an individual can perform a task independently, if the energy cost is so high that it affects the rest of their day, performing the task would be detrimental to their quality of life [44]. While quality of life scores of individuals with SCI are lower than that of the general population $[56,33]$, it is important to note that the disability itself may not decrease an individual's quality of life, so much as the individual's own perception of their ability to cope with their everyday life derived from a combination of factors such as social integration, physical function, and independence [78].

\section{Assistance dog training}

Assistance dogs are trained to support individuals with various disabilities $[2,6]$. The three major classifications of assistance dogs are: guide dogs for individuals with visual disabilities, hearing dogs for individuals with hearing disabilities, and service dogs for individuals with other conditions, such as mobility assistance dogs [74] medical alert dogs [15, 60, 62] , autism support dogs [48], etc. Each type of assistance dog performs a specific set of tasks to support their human owner's needs. A person with tetraplegia may be allocated an assistance dog trained to perform tasks such as opening doors, refrigerators or cupboards, retrieving or picking up objects (e.g. a TV remote or phone), or nudging an individual's limb into specific positions [31].

Positive reinforcement training is an increasingly popular framework for the training of dogs, with many modern training organisations promoting reward-based training $[63,75,1,19]$. Positive reinforcement training involves the presentation of rewards (e.g. treats, vocal praise, toys, petting) in response to desired behaviours. Studies have found reward-based training methods to be associated with higher levels of obedience and fewer problematic behaviours (i.e. aggression, attention seeking or fear behaviours) $[28,84,3]$. Some rewards are more effective than others: importantly, verbal praise alone is not sufficient to maintain performance while tactile stimulation, such as petting, may be [45]. Indeed, while the most effective reward differs between dogs, food-based rewards are shown to be the most effective of all reward categories [23]. However, people with tetraplegia may struggle with many of the reward options, such as petting, giving treats, or playing with toys, and verbal praise may be the only option available to them. The importance of tactile and food-based rewards illustrates some of the difficulties people with tetraplegia may face when attempting to maintain the owner-dog bond.

\section{Benefits of assistance dogs for quality of life}

A growing body of literature investigates the benefits of assistance dogs beyond functional assistance, also considering psycho-social health, well-being and quality of life $[65,61,58$, 79, 64]. Assistance dogs have the ability to positively influence many quality of life factors, such as independence, level of social interaction, confidence, and anxiety and stress levels [83]. Observational studies have found a significant increase in positive social interaction (e.g. smiles and conversation) when individuals in wheelchairs were accompanied by a service dog than when alone [17]. For example, at one mobility assistance dog organisation, $92 \%$ of participants (90\% of all recipients) 
reported that strangers frequently started conversations with them when out in public with their dog, with $75 \%$ stating they had made a new friend since receiving their dog [37]. Other ethnographic studies have used a combination of interviews and questionnaires to reveal that assistance dogs not only increase individuals' opportunities for social interaction, but also increase confidence, communication skills, and positively effect how they are perceived by others [10].

\section{Proposed technologies for people with tetraplegia}

A review of the literature and technologies available on the current market reveal a variety of input modalities and devices to enable people with severe motor impairments to interact with technology. These include mouth-located interaction (e.g. sip and puff and [9]), infrared tracking [7], switches (e.g. [29]), voice [68], and eye-tracking [53]. To further improve the efficiency of these inputs, previous research has investigated new data processing methods which could enable more customisation (e.g. [13]), and built-in software adaptations or custom software [16]. Other work has invited people with a motor disability to speculate on possible new inputs, leading to the development of chairables, wearable technologies for wheelchair users, and found that the space around the arms and the head of the chairs could potentially be used to develop novel forms of inputs [11]. However, there is a gap in knowledge surrounding the uptake and adaptation of such systems by people with tetraplegia.

One area of particular interest within the landscape of technology to support people with tetraplegia is smart-home technology, especially voice activated devices. The increasing adoption of smart assistants, [35] such as Amazon Echo and Google Home, is accelerating the development of voice-operated environments, enabling people with tetraplegia to open doors and manage lights or blinds unaided [47], tasks for which assistance dogs are trained and have previously been relied upon to perform. However, in evaluating the value of assistive smart-home technology to support individuals with tetraplegia, an important consideration is that many novel systems explored by researchers are not evaluated in the field and never reach the market [25]. This is in part due to the cost of deployment as users with disabilities are relatively few, which drives up costs. With technologies that do reach the market, many are discarded as they do not fit within people's everyday life and practices [57]. To respond to this issue, and also to empower people with disabilities as designers, researchers have proposed a focus on Do-It-Yourself Assistive Technologies [32]. Our work described here aims to achieve similar goals: a technically simple implementation that addresses everyday needs and that allows accessible solutions without relying on off-the-shelf products manufactured for small user numbers. The ability to customise technologies, and in particular to allow the use of several types of inputs, is an essential design principle in this area [26].

\section{Design guidelines for canine computer interaction}

An increasing body of work exists where at least one user of the system is a dog (canine) [21]. Indeed, multi-species interfaces, where users of the system are of at least two different species, have been recently developed within an AnimalComputer Interaction (ACI) framework [39]. ACI considers species-specific needs unique to animal users and moves from a user-centred design to involve animals directly in the design process as informants. To do this, many methodologies have been leveraged, such as multi-species ethnography [36, 42] and rapid-prototyping [77, 38, 60, 22]. Interfaces for canine use continue to be developed for different applications for both working and pet dogs, such as computerised pet toys [14], games to facilitate human-dog bonding [81], and interfaces to support the work of medical alert dogs $[60,41]$.

\section{Treat-dispensing devices}

Current off-the-shelf treat dispensing devices are limited in their input modalities for users: they either can only be activated by the button remote that comes with the device (i.e. Treat n' Train [55], Pet Tutor [76]) or more recently, some systems can be voice activated due to their integration with Amazon Echo (Furbo[24], PetCube [54]). However, these voice-activated systems were not designed to be portable, and are all bulky, wall-mounted devices that are not designed to be portable or mounted to wheelchairs. None of these devices adapted to work with different switch devices that are highly variable in different individuals with tetraplegia (i.e. sip and puff, ribbon switches etc.).

In summary, previous research on assistive technologies for people with severe motor impairments or tetraplegia has not investigated how to support, rather than replace, assistance dogs. Additionally, current off-the-shelf treat dispensing devices for dogs are not compatible with different input modalities required by persons using assistive switch devices or that requires wheelchair mounting. This is the gap our work aims to investigate and address.

\section{RESEARCH APPROACH}

We followed a participatory design process. Participatory design emerged from an aim to increase the participation of workers in the design of the technologies they use $[18,71]$. More recently, participatory design has focused on involving marginalised users, both to empower them [46] and to build more adequate technologies [4]. Participatory design is an iterative cycle from exploring and analysing user needs, prototyping, evaluation, and improving the design with the active involvement of participants, as summarised below.

\section{Formative interviews}

We worked with four participants (P1-P4) and two dog trainers (T1, T2) who had trained the assistance dogs (D1-D4) belonging to the participants. Prior to the study, all participants had their own mobility assistance dogs that were trained and supported by the same assistance dog training organisation (located in North America). We wanted to investigate any challenges that the participants were currently facing, specific to tasks their dogs were helping to support them with. To this end, we conducted interviews that explored their daily lives and routines, relationships with their assistance dogs and use of technologies. We analysed these interviews using deductive thematic analysis [5]: the interviews were tagged according to 
the themes we were investigating, which were then compared. We summarised agreements and disagreements between participants and selected the quotes to be included in this paper. This analysis highlighted opportunities for design to support the work of assistance dogs.

\section{Iterative design and prototyping}

We further conducted three design sessions with each participant. Using a participatory design approach for assistive technologies is increasingly considered to be essential to ensure adoption [32]. However, traditional "hands-on" participatory design activities (e.g. drawing sketches or directly prototyping) were not fully appropriate for our participants. Previous research suggested the use of contextual inquiry and methods based on oral communication (e.g. focus groups) for participatory design with participants with limited motor skills $[67,11,7,8]$. Additionally, we were informed by previous work with canine users, that favoured a rapid prototyping methodology to allow as many potential designs as possible to be investigated with users that may have unknown needs (because they are a different species) [60]. Thus, we used a combination of contextual inquiry and rapid prototyping to carry out co-design. During the first design session, we employed a range of materials such as blocks of foam and Velcro to facilitate this process. We asked participants to describe the type of technology they would like to use, with the low-fidelity materials allowing the researcher to quickly demonstrate it to the participants, and collect oral feedback. The trainers also participated with feedback in these sessions. The trainers, also potential stakeholders of the system, provided valuable insight as they were able to advise on the feasibility, usability, safety, and other aspects of the design for both parties (participants and their assistance dogs). Later sessions focused on design critique of the iterative prototype developed.

\section{Evaluation in the wild}

To validate the design, we chose to use a qualitative, inthe-wild approach, in line with participatory design and experience-focused HCI $[34,69]$. The participants were given a working prototype to install on their wheelchairs (the prototypes continue to be used by the participants). We inquired weekly about their use of the prototypes and any incidents which occurred, and participants were also able to contact us at any time if any technical or functional issues arose. We gave technical training to the assistance dog trainers so that they could provide direct system support, since they were considerably more local to the participants than the researchers. This training was similar to that given to the participants when the prototypes were handed over for long-term testing: the trainers were walked through the basic functionality and all features of the physical prototype and the phone app. The goals of this evaluation study were to identify any shortcomings of the prototype and to understand the potential impact on assistance dogs and their relationship with the participants, and through this, the potential of human-canine assistive technology.

\section{Ethics}

There are two ethical challenges particular to this research. Firstly, people with disabilities are largely considered as a vulnerable group necessitating heightened ethical scrutiny. Secondly, as ethics regulatory frameworks largely consider animals as research objects, and not research subjects, there is little oversight on how researchers include and design for them, or that the researchers have sufficient training to be sensitive to their needs [40]. We thus brought together researchers with previous expertise in designing with disabled people and with dogs, who were in a better position to advocate for the research participants (including canine), e.g. through monitoring the length of testing sessions and watching for signs of stress or tiredness. We also relied on the existing practices and framework set in place by the assistance dog training organisation itself, which requires certain practices in regard to the welfare and well-being of the assistance dogs. We also received ethical approval from the C-REC ethics board of the University of Sussex.

\section{FORMATIVE INTERVIEWS}

\section{Use of technology}

We asked the participants about their current use of various technologies in general, and also their current use of any assistive or smart home technology, and/or technology used in relation to their dogs. The participants each used different switches for every day functionality (e.g. urine-bag release, switching modes on their powerchair, and computer input). One participant used a sip and puff switch for primary control of their chair, and had previously also used a ribbon switch installed on the head-rest of the chair. The other participants used a combination of button switches and joysticks. All participants used voice-activated phone control to call for help, if needed. All participants gave us information about their history of adapting voice-activated technologies within their homes. Three of the four participants found that some daily task functionality, that they had previously relied on their assistance dog for, was recently "taken over" by a voice-activation home assistance instead. This was usually related to environmental light control, and the participants still relied on their dogs to open and close doors, cupboards, and drawers, and also to retrieve dropped objects from the floor.

The participants also highlighted two significant limitations of voice-activated home assistants. Firstly, there are currently many limitations to their physical control abilities, which means they cannot replace all of the skills of assistance dogs. Moreover, they also require the installation and maintenance of complex systems, which are often proprietary and are also expensive. In contrast, any new environment (e.g. hotel rooms, offices, a friend's or family member's home) can fairly quickly and inexpensively be adapted to enable an assistance dog to help. This is because, rather than installing motorised systems, "dog adapting" an environment requires only securing ties on handles. These spaces cannot be completely adapted, but are can be made significantly more accessible.

\section{Relationships with assistance dogs}

A common theme among all the participants was frustration with reinforcing their dog's existing training, due to being unable to independently feed (treat) their dog. Indeed, some participants recalled initial anxiety about getting their first 


\begin{tabular}{ccccc}
\hline Participants & Treat Ability & Dog Breed & Powerchair & (Phone)App use \\
P1/D1 & None & Labrador & Quantum Edge & None \\
P2/D2 & Partial & Goldador & Quantum Edge & High \\
P3/D3 & Partial & Labrador & RangerX & High \\
P4/D4 & None & Golden Retriever & NuMotion & Some \\
\hline
\end{tabular}

Table 1. Study participants and their ability to treat their dog, the dog's breed, type of powerchair they own, and their ability to use apps on smartphones.

assistance dog, and the worry of how they would care for the dog: "When I first got injured, those was my thoughts- could I feed him properly, get him water? Especially for my first one, he listened and he helped. Him being willing to drink water out of a cup, for example, helped, but I know not all dogs can [do that]." (P3). Other participants reflected similar initial concerns or frustrations. Once a dog had been matched to them and placed with them, all participants went through an initial intensive training and bonding "bootcamp" period in order to solidify the partnerships. All the participants were initially supported in treating and feeding their dogs during their initial matching and training phase: trainers would place the dog's food, in a bowl, physically on the individuals' lap so that the dog would build a positive association with them. One participant reflected, "[At bootcamp] I pretty much got a bag, a little bag, and they wanted me to try to stick my hand in there, and that just didn't work for me. I was thinking at some point this needs to get better - I need to find some better way to give this guy a treat." (P3).

We learned about each participant's current practice and abilities in independently treating their dog for maintenance training (that is, long-term reinforcement for continued bonding and motivation after the initial learning of skills). While three of the participants could not treat their dogs completely independently, one of the four participants was able to treat his dog independently with a very particular system that he had devised through trial and error: "I use my mouth to get the treat, because I can't grip with my hand." (P2). P2's system required the both of use his hand and mouth to balance a treat on side of one hand after getting a treat out of the bag using his mouth. While P2 was alone in being able to treat his dog completely independently, he did note that it was not a fast process and very limited in what treats he could use because of the involvement of his own mouth: "This thumb always tucks in. So the treat would just fall right through on that hand. It took a lot of experimentation to find the right brand of dog treats to work with this system. I didn't like the soft treats because then I'm tasting. I don't want the treats that are too flaky, again, because then I'm eating some of them!" (P2).

The other three participants were reliant on another person to treat their dog for them. Two of these participants were still able to pet their dog for positive reinforcement, though the other participant could not easily stroke or pet her dog at all, as it would require moving her hand off her armrest and she was unable to re-position it. Although her dog was trained to nudge her hand back on the armrest, this was quite risky to do when she was completely alone, without someone else present to help if needed. Significantly, this participant had previously been rejected by several assistance dog training organisations to be placed with a dog because of the severity of the challenge of placing a dog with a person that could not use pet or food reinforcement directly. The organisation in this study was the first to take on this challenge, and the partnership had turned out to be successful. However, this is in part due to her relying on carers to place a treat on a tray mounted on her chair on her behalf. P1 would then give a verbal release to the dog, to create an association between her and the treat, and lessen the association between the carer and the treat. However, P1 still experienced frustration and challenges with this system: "We were together the other day and he knew that the treat was going to be coming from her, so even though I said 'yes', he looked at her and looked back at the tray and then her again." (P1). P3 voiced issues with their current practice as well, saying: "He's not always going to expect a treat every time I drop something and he retrieves it. But sometimes I do need/want to treat him and I just simply can't. The gripping can be tiring. Just trying to do that pinch between that thumb, that wears you out trying to do that...you can't just reach down and get the treat. So then you get frustrated. Sometimes you just drop it." (P3)

\section{Proximity and importance of food reward}

After initially identifying a theme of anxiety and challenges around feeding their dogs, we followed-up with the trainers and asked them about the nuances of proximity and association with food. The trainers confirmed the importance of treating for the bonding with an assistance dog: "I do find as a trainer that it does make a difference in the quality of the experience for the dog to vary the type of treat offered day to day. And you know the dog is like 'what's in there, what am I getting now?'" (T1). They also shared their thoughts on the importance of the way of treating: "It helps the bonding and helps encouraging the bonding when the dog sees their person as the specific resource. Treats coming out of machines on their own are acts of god. Treats coming out near their person once their person has verbally marked it should be an act of their person." (T1). The treat must come from very near the person, when the dog can smell both the person and the treat: "If right by the leg the dog can see and smell your leg, that's surely a stronger association than if it was on the back of the chair." (T2)

We also found that trainers have tried many different interventions to facilitate treating. This included DIY prototypes and teaching new skills to dogs: "We tried to use a tube delivery system where a pate in a stick could be squeezed out for the dog. We thought they could use their arm to press against armrest against the tube to squeeze out the pate to treat the dog. But some of our clients couldn't do it." (T1).

\section{ITERATIVE PARTICIPATORY DESIGN PROCESS}

Following our initial interviews, we used an iterative participatory design process to create an accessible treat dispenser that 
can be mounted on the side of a wheelchair, thereby enabling people with tetraplegia to give food rewards to their dog to facilitate bonding and training. The system was developed in three phases.

\section{Phase 1: Early design sessions}

Having identified the need for a highly customisable system, we worked with participants to further define the form factor. To facilitate this, during the early design sessions, we prompted participants to describe what a tool that would help them reward their dogs might look like. As mentioned above, participatory design methods such as asking participants to sketch or form prototypes were not appropriate in this study, so we relied on a mixture of verbal descriptions and rapid prototyping (i.e. researchers rapidly fashioning potential solutions out of foam in-situ to obtain feedback).

\section{Participants' descriptions of their ideal device}

Participants' descriptions of their ideal device included both form factor and setup requirements, concerns for their dog's well-being, and design considerations to support their autonomy and their bonding with the dog. Participants suggested designs similar to "PEZ dispensers" (P3) or designs with an automatic "little door" or "like a little slide into a little cup" (P1).

Form factor and mounting: Using blocks of prototyping foam and an example treat dispenser (purchased off-the-shelf, marketed to dog trainers without upper-limb disabilities) enabled us to define with participants the size of the device and its placement, as well as requirements specific to the assistance dogs. We found that the treat dispenser has to be small enough to strap on the side of the wheelchair (maximum size 6" $\mathrm{x} 4$ " x 3") and not extend the space profile of the wheelchair in any direction. Additionally, it has to especially robust as it may take sudden motion hits or be knocked by a carer or dog. These sessions actually further revealed the general desire of participants to be able to customise their wheelchairs, and the limitations they currently experienced in this regard: "They have very little in the way of things to mount on. They're very poorly designed. They're not customisable..I've had to have my chair's arm-rest cut off and re-welded to have them raised up higher." (P4). Moreover, as participants wheelchair setups varied considerably, even among participants with the same wheelchair, the mounting mechanism needed to be highly flexible - the participants suggested zip-ties. As phrased by P1: "Zip-ties are actually good. They work.". P3 also told us that they "always carry zip-ties in our bags. They are used so much in general. But with this device, I mean you wouldn't want take it off and on every single day. But it should be easily taken off if necessary, [which a zip-tie is good for]. Because we are often going out of our chair or moving it around to recline."

Power: Optimally, the treat dispenser would be USB powered because it would require too much labor to dismount, charge and remount the device daily. Participants pointed out that new power wheelchairs usually have on-board USB, and that they also have USB power packs as an alternative power source: "This chair does not have USB charging on it. ...It is basic in the extreme. But I have a wireless charger that I can set my phone on. But my new chair will come with a built-in USB" (P4).

Device control: Participants varied in how they envisioned controlling the device, with some interested in potentially using the Bluetooth controller on their chair, and others picturing the dispenser controlled by a phone app. This was consistent with the systems the participants currently used to control various functionalities. As there was no clear preference in terms of the method for controlling the device, we followed the requirements of high adaptability we had identified during the formative interviews.

\section{Dispense receptacle and treat capacity}

One requirement particularly emphasised by all participants was for the device to dispense into some sort of a tray or receptacle (i.e. not free falling out onto the floor). Both the participants and trainers emphasised that an assistance dog eating anything off of the floor goes against the training of most assistance dog frameworks. This is because assistance dogs are expected to be able to go into cafes, restaurants, or public places where there could be food on the floor, and understand that they should not attempt to eat anything from the floor. As many dogs would naturally prefer to scavenge or eat accessible food, this has to be a specifically trained behaviour, usually early on in an assistance dog's training, and part of supporting this training is ensuring the dog is never allowed to eat freely off the floor. Moreover, the requirement was also considered important for bonding: "it could be on their chair but the delivery we don't want it on the floor, because that encourages scavenging. It just has to be in proximity to your body to the point where the dog doesn't say floor treat, the dog says dad treat" (T1). This, however, is not the case of most electronic treat dispensers on the market. Finally, there is a requirement that the dispenser has the capacity to hold enough treats for the individual to be able to have a meaningful training session or treat throughout a several hour period where there may be no carers, co-workers, friends or family around to help them re-fill the device. The desired treat capacity varied slightly across participants, but all wanted at least five treats, or as many as ten, which is the most they thought they might use on any given day during a period when they are alone with their dog (without anyone there to help refill it). Said one participant: "I give her about 8 treats a day, 8 different times, one treat at a time. When on occasion I want to give her several treats at a time as a 'jackpot'. What I do is, I'll put like four or five in my mouth then one at a time spit them out." P2. Another participant reflected on their treat frequency: "I would say with me working, once an hour. That's what I do now. I'm not gonna sit up and give him every 10 minutes or even 30 minutes. Maybe every hour to keep his attention and..[less bored] ..while I'm working. When I was new partnership they had me trying to give him treats basically everything he did. But now like I say 8 or 9 times a day." (P3).

\section{First prototype}

Form factor and fabrication: Many dispensers use retracting doors to reveal the treat ${ }^{1}$. However, in order for this mech-

\footnotetext{
${ }^{1}$ Ready Treat V2.0 Radio-Controlled Treat Dispenser: Users activate the remote to open the door to the treat compartment to reveal a treat
} 


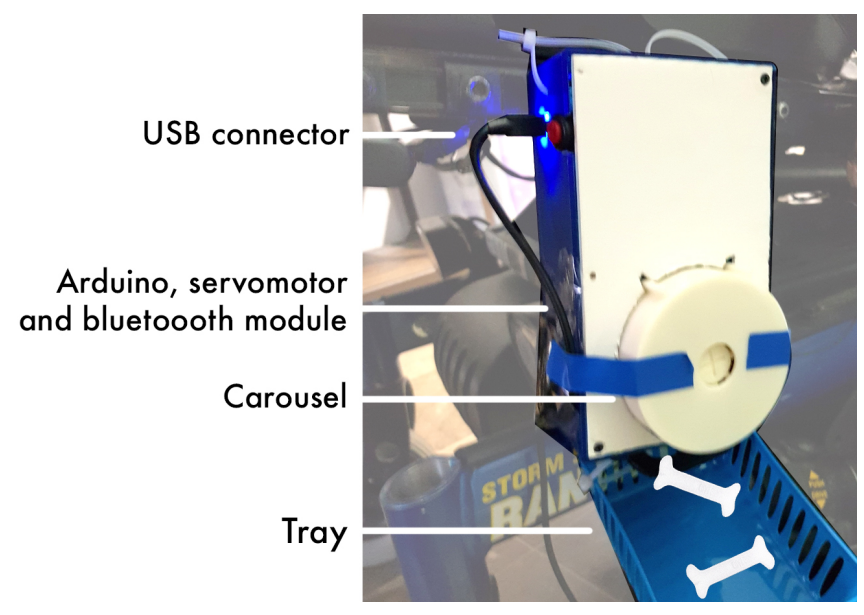

Figure 2. First working prototype consisted of a vertical rectangular box with a plastic tray attached at the bottom where dogs can collect their treats. The box features a carousel into which treats can be loaded. The electronics are placed at the back of the box, behind the carousel, as is the USB connector

anism to work, there needs to be a large enough receptacle for the dogs nose and mouth to fit in order to access the treat. In non-wheelchair versions of treat dispensers, space is not an issue, but with this system, we needed to provide space for the dog's nose and mouth to fit (the dog eating the treat off of the floor was not an option) within the given size constraints. Thus, we decided to use a rotating carousel with separated sections each holding a treat. With each activation, the carousel rotates one section forward, releasing a treat through a gap at the bottom of the carousel. A model of the internal mechanism (carousel, etc.) was made using Tinkercad, an online 3D modelling tool, and then 3D printed. The remainder of the prototype was made with a variety of materials (cardboard, plastic tray), to encourage participants' feedback [20].

Hardware: Our initial prototype consisted of a carousel operated by a servomotor connected to an Arduino Micro (a small micro-controller board), powered via USB connector. The Arduino Micro allows for rapid prototyping as it is compact and designed to easily slot into a solderless breadboard. As we could not guarantee reliable $\mathrm{Wi}-\mathrm{Fi}$ connections outdoors, we opted for Bluetooth Low Energy for communication with the treat dispenser app, due to it's low power usage. The rotation of the carousel was controlled by a continuous rotation servo, and the rotation amount controlled by applying a "run" signal to the motor for the time taken to rotate one section (around 200ms). When the Bluetooth module received a "dispense" signal from the app, or from a user's own control switch, the Arduino would rotate the servo one section, dispensing a treat.

Software: We developed an Android smartphone app to control the treat dispenser and manage user preferences. The app was optimised to work with Android's inbuilt accessibility options and could also work in tandem with the users' own control switches. After it's first use, the app could pair automatically with the treat dispenser. The software has three main functions: (1) Dispense treats (2) Reload the dispenser (which reset the number of treats to the dispenser capacity) and (3) Set user preferences. A control screen indicates the remaining number of treats and battery level, and there are preferences screens for both the app and treat dispenser device. Preferences included selecting audio feedback on the app and dispenser (for treat dispensing, last remaining treat, dispenser empty). The audio feedback could be set to a variety of sounds or completely disabled.

\section{Phase 2: Design critique}

We presented the first prototype to the same group participants in design critique sessions. We invited them to participate in a design critique of the prototype to support further iterations.

\section{Participants' design critiques}

These design critiques confirmed the importance of the adaptability to a wide range of inputs: two of the participants were affirmative they only wanted to use a physical switch. Said P1 "As I was saying before, I cannot actually control any of the apps on my phone. That's awesome you can plug in the different switches."). Unfortunately, not all of the functionality implemented on the phone app was available to users that preferred to use only a switch, something that did not go unnoticed in the critique. For example, P3 asked about the phone app's ability to display how many treats remained: (P3: "Could that feature be put on a button instead? Like a long-press and different beeps? And you can hear the beeps to let you know how loaded it is?"). Two participants wanted the option to use both mechanical switches and the app, and potentially other inputs in the future: they wanted to see what might work for them, and since they did use phones on their app sometimes, were interested in comparing which would work better for them (controlling from their phone or from a physical dedicated switch).

The design critiques focused on usability and form factors issues:

- Feedback: to phase treat giving (until the device can be reloaded), there needs to be feedback about how many treats are remaining $(\mathrm{P} 1, \mathrm{P} 2, \mathrm{P} 3)$.

- Calibration and larger capacity hopper: due to the desire to accommodate a variety of textured treats, this needs to be easily adjusted for treat variety. They also flagged the need for an interchangeable tray system for different users, owing to different dogs' behaviour, snout size/height, as the dog's angle coming at tray changes ease of use.

- Easier to load with treats, clear (see-through) lid (user can see in mirror, everyone else can see directly). The most significant design flaw highlighted was that the initial functional prototype required someone other than the participant to tell the system to 'reload'. Our initial functional design had an external button on the device that needed to be pressed when the dispenser was re-loaded. (Note: this functionality was available in the phone app, but as discussed some participants did not use phone apps at all, and would only be using the system through an external switch.) "I'm just gonna be honest with you-some attendants forget those small tedious things. And it happens to me all the time. And I wouldn't' want them that they would have to remember to 
push a button on here. I would rather that be MY responsibility." (P3). "If my carer forgets and leaves, so I can't reload it?" (P1).

- Positioning "I'm kind of thinking- depending on the size it ends up- maybe it could go between my feet?". (P2). In considering the mounting placement of the device, there was potential tension between flexibility in placement and safety of the dogs if a front placement (between legs/feet) were to cause the dog to move in front of a moving chair in anticipation of a treat, potentially being injured. This was discussed with the dog trainers we worked with, who highlighted that the training framework teaches dogs not to go in front of the wheelchair while moving and also for the person to not reward mark OR to treat a dog while the chair is moving. So in practice, neither of these things should occur, and the dog should thus not be motivated to think any treat will ever be dispensed in-movement. However, we can see how a dog might still, especially in the early stages and when the device is novel to them, dart in front of the chair, so we highlight this issue here. "You also have to think, I'm usually in this position, but I can fully recline. How's it gonna fit then? Is it gonna hit my light?" (P3).

\section{Dogs' interactions with system}

Observing the dogs' reactions highlighted the tensions between humans' and dogs' needs. For instance, there was tension between the amount of space required for a dog to fit their snout and the amount of space available on the chair; and between the need to capture the treat securely and ensure it did not go flying out of the tray, with the need avoid having a restricted 'corner' that the dog struggled to get the treat out of. Our initial designs did not anticipate how much difficulty the dogs would have in reaching the treat if it was in a corner of the tray. Some dogs, at some tray angles, had to tilt their heads and lick to get to the treat. However, the dogs also demonstrated a rapid understanding of the interaction: "He knows already that it will come out after the reward marker. That's why he's standing right by it now. Its a magic little box" (P1).

\section{Second prototype}

Form factor and fabrication: The most important changes in form factor were that we made every aspect of the device more customisable. We modeled and 3D printed carousels with different treat capacities, allowing users to choose their preferred capacity and treat size. We also separated the tray from the treat dispenser and a new design was created which hooked onto the outside of the main module so users could detach and move the tray flexibly (see Fig. 3). In this way, the tray could also be made in different sizes and shapes. All parts of the device were 3D printed.

Hardware: As some of our participants preferred using the device without the app, and all had highlighted the importance of having control of the 'reload' function, the reload button was removed from the dispenser and the reload control was changed to a long press of the user's external switch.
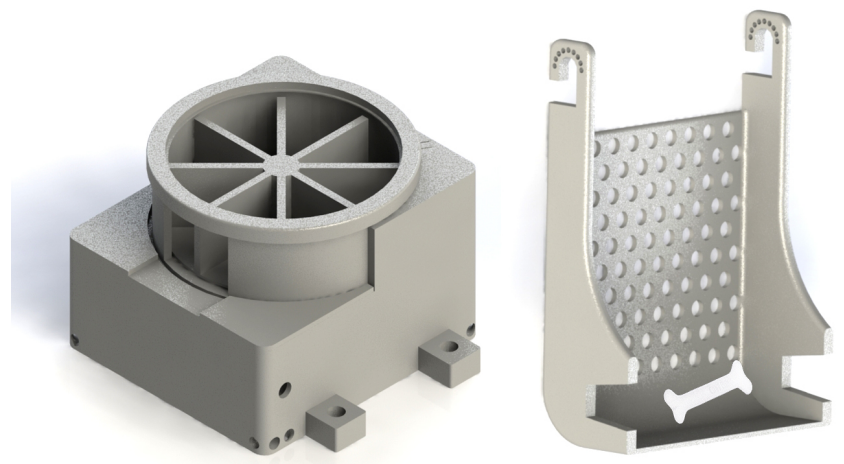

Figure 3. 3D model of the second prototype: the two-parts (automatic carousel and attachment, and tray) can be fit together and attached to a wheelchair using cable ties

\section{Phase 3: On-going usability evaluation in the field}

During the third phase of the design process, we provided the prototype to our participants and once again engaged them in a longitudinal critique.

This evaluation revealed that the rotation amount of the carousel was inconsistent (i.e. under- or over-rotating) due to variations in factors such as weight and supply voltage, requiring a manual offset for the servo timing. Although this could, in principle, be set in the app, this wasn't a practical option for the participants and so another solution was required. Also, that strengthened tray connection points would be required for increased robustness as the dogs used varying force in retrieving treats and the device had to withstand rough handling from carers unfamiliar with the device.

\section{Third prototype}

The use of timed rotation proved to be too unreliable due to variations in USB power (the standard allows for a 5\% voltage variation), and variations in loading from frictional effects of the moving parts and the changing weight of treats as they were dispensed. Our solution was to use a microswitch activated by raised location markers at the bottom of the carousel to indicate when the carousel had rotated by the required amount.

Form factor and fabrication: The raised notches on the bottom of the carousel identified the segment walls and the servo would turn until a notch activated the microswitch, meaning the carousel would always be rotated by the correct amount regardless of weight or voltage, simplifying the entire system. The connection point between the main dispenser and the tray was also strengthened by utilising a nut and bolt mechanism rather than 3D printed hooks.

Software: Factors such as voltage and weight were no longer significant, so the manual offset function could be removed from the app.

\section{Impact on relationship}

We were given feedback from participants on the impact that having the device mounted on their chair had on their rela- 


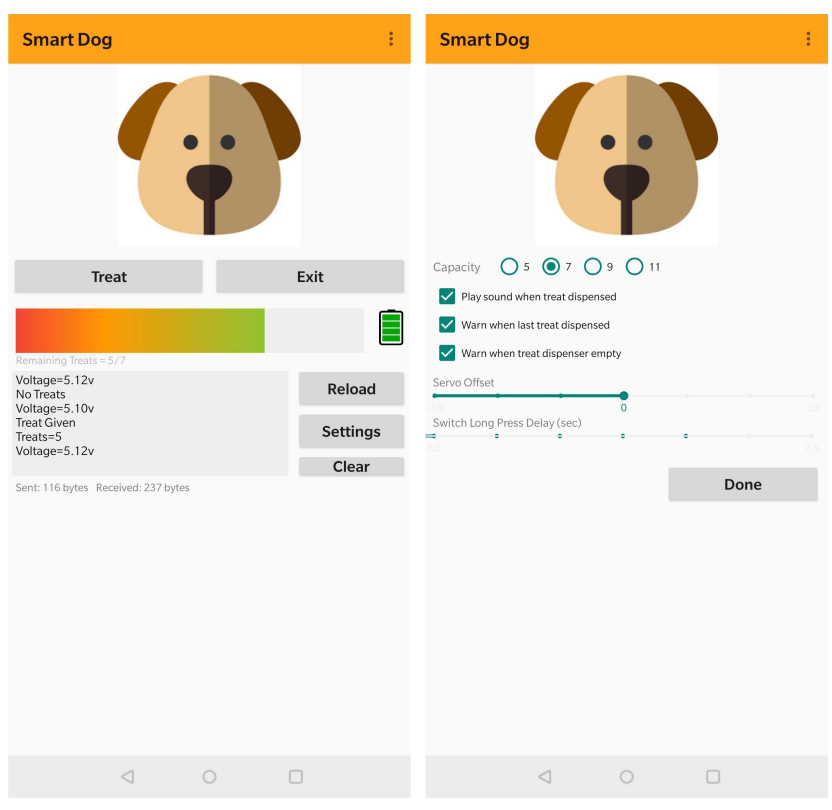

Figure 4. Screenshots of app showing (L) the main screen from which users can dispense a treat, exit the app, reload the dispenser and open settings, and ( $R$ ) the settings screen where users can control variables such as treat capacity, offset and sound

tionship with their dog. The feedback in this area was overwhelmingly positive: all participants said that it had changed the relationship they had with their dog in a positive way. For P1, the participant that had the most limited ability to interact with her dog (because she could not provide petting reward in addition to food reward without the intervention), she reflected that "it's just really cool. He's so smart and he knows exactly what's going on. He doesn't look to anyone but me when there's a verbal marker cue."

For the other three participants $(n=3)$, that were unable to independently treat their dog prior to the deployment of the device, they reported perceived changes in how much attention their dog gave them and excitement in the dog in getting to interact with something new:"There's a big difference to how he responds to me". And P3: "You know I think that was pretty cool especially how D3 responds to me and treats I think that was pretty cool how he followed me and stayed. It changed his personality. I can see him looking for someone else to treat him and [now I can] be 'like no I got you'."

\section{FINAL DESIGN}

The final treat dispenser consists of a 3D printed outer case and inner carousel, a micro-controller, and several electrical components as shown in [Fig. 1]. It can be mounted on, or detached from, the side of a wheelchair and connects via USB cable to a wheelchair's in-built USB port. The friction between parts is minimised and when the front panel is closed, the dispenser is waterproof, protecting the electronics. Users of the device, including carers, can interact with the app or via a $3.5 \mathrm{~mm}$ jack, compatible with the users' switch of choice, to activate the treat dispensing.
The participants were given a fully functional working prototype for testing. The deployed prototype was a second iteration on our initial design attempt. The prototypes allowed participants to independently treat their dogs, without requiring a carer or other person to be in the room. The participating dogs learned that the dispenser provided food from testing during initial design critique sessions. Over time, the dogs appeared to make a connection to their owner's verbal cue and the dispensed food, demonstrating an expectation that a treat was about to dispense when the verbal marker occurred. All prototypes were configured to make different sounds for different states of the device, including upon dispensement of a treat. Further work should explore the connection and importance between the owner's verbal ques, verbal reward markers, the sound that the devices make, and how this should or could be integrated into existing training frameworks.

Any initial displays of over-interest in the device, or behaviour consistent with the expectation of arbitrary dispensing, faded once the device was deployed and consistently used. As a result of being able to provide food reward independently, without any assistance from another person, participants reported feelings of empowerment and excitement, and indicated that long-term use of such a device could have potential significant positive impact on their relationship with their dogs.

\section{DISCUSSION}

\section{Designing with Assistance Dogs and their Human Com- panions}

With this work, we aimed to highlight the potential for assistive technologies to support the work of assistance dogs - instead of attempting to replace them (e.g. with robotics). This work provided an initial exploration of some of the challenges that persons with tetraplegia and their assistance dogs face, and sought to technically intervene to address one such problem, that of challenges with independently giving food rewards to their dogs. However, over the course of this study, many other potential areas for intervention were also highlighted by participants and dog trainers alike. These include investigating how technologies could encourage play and exercise for assistance dogs (such as accessible ball launchers), enabling dogs to interact with smart home technologies, and technologies to support the relations between children and their autism-support assistance dogs. This is an example of disability Interaction [30]: the case study opens new perspectives for the design of home technologies more generally.

We demonstrate with our case study that dogs can be actively involved in the design process by using simple materials and low-fidelity prototypes. This allows designers to observe dogs' reactions, which, in tandem with stakeholders and experts present (e.g. both trainers or dog specialist and dog companions), is preferable to understand what the dog is responding to. As there are no guidelines on ergonomics for assistance dogs, future investigations could more precisely develop the boundaries of this design space, such as systematically investigating the placement of systems on a wheelchair.

An issue noted in previous research (e.g. [11]) is that working with people with tetraplegia benefits from repeated engage- 
ments. This is both helpful to empower [32] participants in feeling confident they can invent and describe the systems they need and because their confidence increase with time, which eases the partnership with dogs and researchers. There is always the risk that the needs of people with more experience with technologies and design processes are better catered to, but this work moves from the assumption that such research is necessary to help foster communities of disabled experts in design [80, 32].

Finally, this type of design approach could support interdisciplinary research, in particular dog cognition and social behaviors. Initial use of the prototype suggests assistance dogs do indeed associate treat delivery with the human closest in proximity, or using a verbal reward marker. Further exploration of this relationship would be useful and potentially facilitated with further applications of Canine-Computer Interaction.

\section{Implications for design}

\section{DIY-AT: Customisable Design}

This work is informed by previous work on Do-It-Yourself assistive technologies where the goal is to empower users with unique needs without reliance an expensive devices. Humancanine interaction and canine-computer interaction had not previously been investigated within this paradigm, but moving forward should be considered. It is worth nothing that although we had envisioned at the beginning of the project that we would need to customise the device to different wheelchairs and wheelchair users, we had not anticipated customisation for dogs and their personalities. Future work on this topic should bring together members of the assistive technology and the canine-human interaction community. We did not investigate, for instance, existing practices of DIY technologies for dogs, but our formative work suggest these initiatives exist.

Universal Adaptable Design: Open Hub for accessible inputs This work also highlights a need for an open platform enabling the use of a range of inputs with electronic systems. This would be consistent with the aims of the Xbox adaptive controller, though this is not usable by researchers outside of the Xbox platform. In line with efforts to bring about less expensive, more open and customisable assistive technologies, all of the files and instructions necessary to reproduce this device and, importantly, enable the use of multiple inputs, are available online (https://github.com/alice-t/Treat-Dispenser). We argue that the development work on the platform to connect to the treat dispenser could open new opportunities for other devices for people with motor disabilities. Thus we intend for this work to echo the principles of universal design, which includes offering a range of ways of operating a given device [73]. This could provide a new design space for DIY-AT projects.

\section{Embodiment: potential of chairables}

Chairables had previously been investigated as a new type of inputs, but Carrington et al. [11] pointed out how, by modifying wheelchairs, we might affect the sense of embodiment of their users. The ways wheelchairs become part of identity and embodiment has long been investigated in disability (e.g. [50]). Interestingly in our study, some participants suggested they had very negative perceptions of their wheelchairs, which could not be customised or were not performing as well they hoped. Working with wheelchair users and assistance dogs has the potential to enrich how we can investigate these questions: did the dogs recognise the treat dispensing as an action done by their human companion? Could this modify, not just the relationships with their dogs, but also their own perceptions of the wheelchair and its efficiency?

\section{Limitations and future work}

This work has provided an initial exploration into the needs and practices of a user group that includes both humans and dogs and their relationship with each other, that had previous not been explored. Note that we do not expect our findings here to generalise to all persons with tetraplegia with assistance dogs, but rather to open a new design space to investigate. Even from this perspective, this study has limitations: for example, we worked with participants receiving services from the same organisation, which can lessen the variety of answers received. It is possible that other frameworks for training dogs or cultural differences in the status of assistance dogs would expose different practices or needs. However, for the individuals in this study, and others like them in the assistance dog community, there is potential for future further exploration and technological intervention.

In addition to the perspectives outlined above, it would be beneficial for future work to develop and test toolkits for such projects. These could consider different cultural contexts, and different types of assistance dogs, or the same type of assistance dogs within different training frameworks. Moreover, future work could focus on more quantitative evaluations, which would support framing these devices as accessibility aids that could be covered by governmental funding. This could focus on: time to treat if deployed with users that could previously independently treat their dog (but slowly or in a limited fashion); comparing different input modalities (such as the introduction of a customised switch, voice activation, etc); comparing different type of feedback configurations in relation to dogs interaction with device, etc.

\section{CONCLUSION}

We have examined the potential for technological intervention in a specific application at the intersection of accessible technology and canine computer interaction. We have determined there is a gap in research work that considers appropriate methodology and design solutions for individuals that have limited use of extremities, but have the need and desire to bond with their dog and increase their quality of life. We have created a solution through an iterative design process that has successfully been used by four individuals with tetraplegia and their dogs, with positive results.

\section{ACKNOWLEDGEMENTS}

This work has received funding from University of Sussex Research and Development Fund (Round 10). We would like to thank all of our anonymous participants (human and nonhuman) for their collaboration. 


\section{REFERENCES}

[1] California Service Dog Academy. 2019. About Us. (2019).

https://californiaservicedogacademy .org/about-us/

[2] Hilary M Audrestch, Chantelle T Whelan, David Grice, Lucy Asher, Gary CW England, and Sarah L Freeman.

2015. Recognizing the value of assistance dogs in society. Disability and health journal 8, 4 (2015), 469-474.

[3] Emily J Blackwell, Caroline Twells, Anne Seawright, and Rachel A Casey. 2008. The relationship between training methods and the occurrence of behavior problems, as reported by owners, in a population of domestic dogs. Journal of Veterinary Behavior 3, 5 (2008), 207-217.

[4] Simon Bowen. 2010. Critical theory and participatory design. In Proceedings of CHI. Citeseer, 10-15.

[5] Virginia Braun and Victoria Clarke. 2006. Using thematic analysis in psychology. Qualitative research in psychology 3, 2 (2006), 77-101.

[6] Annika Bremhorst, Paolo Mongillo, Tiffani Howell, and Lieta Marinelli. 2018. Spotlight on Assistance Dogs-Legislation, Welfare and Research. Animals 8, 8 (2018), 129.

[7] Emeline Brulé. 2016. Playing Music with the Head. In Proceedings of the 18th International ACM SIGACCESS Conference on Computers and Accessibility. ACM, 339-340.

[8] Michael Bui. 2019. Empathic Techniques for Co-Design with Spinal Muscular Atrophy Type II. B.S. thesis. University of Twente.

[9] Héctor A Caltenco, Lotte NS Andreasen Struijk, and Björn Breidegard. 2010. TongueWise: Tongue-computer interface software for people with tetraplegia. In 2010 Annual International Conference of the IEEE Engineering in Medicine and Biology. IEEE, 4534-4537.

[10] Mary Michelle Camp. 2001. The use of service dogs as an adaptive strategy: A qualitative study. American Journal of Occupational Therapy 55, 5 (2001), 509-517.

[11] Patrick Carrington, Amy Hurst, and Shaun K Kane. 2014. Wearables and chairables: inclusive design of mobile input and output techniques for power wheelchair users. In Proceedings of the SIGCHI Conference on Human Factors in Computing Systems. ACM, 3103-3112.

[12] Marie Chan, Eric Campo, Daniel Estève, and Jean-Yves Fourniols. 2009. Smart homes-current features and future perspectives. Maturitas 64, 2 (2009), 90-97.

[13] C Chandramouli and Vivek Agarwal. 2009. Speech recognition based computer keyboard replacement for the Quadriplegics, Paraplegics, paralytics and amputees.
In 2009 IEEE International Workshop on Medical Measurements and Applications. IEEE, 241-245.

[14] CleverPet. 2019. CleverPet: Exercise your pet's mind and body. (2019). https://clever.pet/

[15] Deborah J Dalziel, Basim M Uthman, Susan P Mcgorray, and Roger L Reep. 2003. Seizure-alert dogs: a review and preliminary study. Seizure 12, 2 (2003), 115-120.

[16] Pete Denman, Lama Nachman, and Sai Prasad. 2016. Designing for a user: Stephen Hawking's UI. In Proceedings of the 14th Participatory Design Conference: Short Papers, Interactive Exhibitions, Workshops-Volume 2. ACM, 94-95.

[17] Jane Eddy, Lynette A Hart, and Ronald P Boltz. 1988. The effects of service dogs on social acknowledgments of people in wheelchairs. The Journal of psychology 122, 1 (1988), 39-45.

[18] Pelle Ehn. 1988. Work-oriented design of computer artifacts. Ph.D. Dissertation. Arbetslivscentrum.

[19] The Guide Dogs for the Blind Association. 2019. Training With A Guide Dog. (2019). https://www. guidedogs.org.uk/services-we-provide/guide-dogs/ training-with-a-guide-dog\#how-are-guide-dogs-trained

[20] Christopher Frauenberger, Julia Makhaeva, and Katharina Spiel. 2017. Blending methods: Developing participatory design sessions for autistic children. In Proceedings of the 2017 Conference on Interaction Design and Children. ACM, 39-49.

[21] Larry Freil, Ceara Byrne, Giancarlo Valentin, Clint Zeagler, David Roberts, Thad Starner, Melody Jackson, and others. 2017. Canine-centered computing. Foundations and Trends ${ }^{\circledR}$ in Human-Computer Interaction 10, 2 (2017), 87-164.

[22] Fiona French, Clara Mancini, and Helen Sharp. 2016. Exploring methods for interaction design with animals: a case-study with Valli. In ACI'16: Proceedings of the Third International Conference on Animal-Computer Interaction. ACM.

[23] Megumi Fukuzawa and Naomi Hayashi. 2013. Comparison of 3 different reinforcements of learning in dogs (Canis familiaris). Journal of Veterinary Behavior: Clinical Applications and Research 8, 4 (2013), 221-224.

[24] Furbo. 2019. Furbo Dog Camera UK I Treat-tossing Pet Camera with HD Cam, 2 Way Audio Barking Alert. (2019). https://shopuk. furbo. com/

[25] Monica Gori, Giulia Cappagli, Alessia Tonelli, Gabriel Baud-Bovy, and Sara Finocchietti. 2016. Devices for visually impaired people: High technological devices with low user acceptance and no adaptability for children. Neuroscience \& Biobehavioral Reviews 69 (2016), 79-88. 
[26] Peter Gyory, Clement Zheng, Daniel Leithinger, and Ellen Yi-Luen Do. 2019. HOT SWAP: Probing Embodied Game Interfaces With Reconfigurable Controllers. In Companion Publication of the 2019 on Designing Interactive Systems Conference 2019 Companion. ACM, 183-187.

[27] K Whalley Hammell. 2004. Exploring quality of life following high spinal cord injury: a review and critique. Spinal cord 42, 9 (2004), 491.

[28] EF Hiby, NJ Rooney, and JWS Bradshaw. 2004. Dog training methods: their use, effectiveness and interaction with behaviour and welfare. Animal Welfare 13, 1 (2004), 63-70.

[29] Steve Holburn, Dong Nguyen, and Peter M Vietze. 2004. Computer-assisted learning for adults with profound multiple disabilities. Behavioral Interventions 19, 1 (2004), 25-37.

[30] Catherine Holloway. 2019. Disability interaction (DIX): a manifesto. interactions 26, 2 (2019), 44-49.

[31] Geoffroy Hubert, Michel Tousignant, Fran Routhier, Helene Corriveau, and others. 2013. Effect of service dogs on manual wheelchair users with spinal cord injury: A pilot study. Journal of Rehabilitation Research \& Development 50, 3 (2013).

[32] Amy Hurst and Jasmine Tobias. 2011. Empowering individuals with do-it-yourself assistive technology. In The proceedings of the 13th international ACM SIGACCESS conference on Computers and accessibility. ACM, 11-18.

[33] Mikko Kannisto, Juhani Merikanto, Hannu Alaranta, Hannele Hokkanen, and Harri Sintonen. 1998. Comparison of health-related quality of life in three subgroups of spinal cord injury patients. Spinal cord 36, 3 (1998), 193.

[34] Joseph 'Jofish' Kaye. 2007. Evaluating experience-focused HCI. In CHI'07 extended abstracts on Human factors in computing systems. ACM, 1661-1664.

[35] Bret Kinsella and Ava Mutchler. 2018. Smart speaker consumer adoption report. Voicebot. ai March (2018).

[36] S Eben Kirksey and Stefan Helmreich. 2010. The emergence of multispecies ethnography. Cultural anthropology 25, 4 (2010), 545-576.

[37] DR Lane, June McNicholas, and Glynn M Collis. 1998. Dogs for the disabled: benefits to recipients and welfare of the dog. Applied Animal Behaviour Science 59, 1-3 (1998), 49-60.

[38] Jacob Logas, Will Mitchell, Monira Khan, Lorita Freeman, Clint Zeagler, and Melody Moore Jackson. 2018. A toolkit for animal touchscreen slider design. In Proceedings of the Fifth International Conference on Animal-Computer Interaction. ACM, 13.

[39] Clara Mancini. 2011. Animal-computer interaction (ACI): a manifesto. interactions 18, 4 (2011), 69-73.
[40] Clara Mancini. 2017. Towards an animal-centred ethics for Animal-Computer Interaction. International Journal of Human-Computer Studies 98 (2017), 221-233.

[41] Clara Mancini, Rob Harris, Brendan Aengenheister, and Claire Guest. 2015. Re-centering multispecies practices: a canine interface for cancer detection dogs. In Proceedings of the 33rd Annual ACM Conference on Human Factors in Computing Systems. ACM, 2673-2682.

[42] Clara Mancini and Jussi Lehtonen. 2018. The Emerging Nature of Participation in Multispecies Interaction Design. In Proceedings of the 2018 Designing Interactive Systems Conference. ACM, 907-918.

[43] Clara Mancini, Sha Li, Grainne O'Connor, Jose Valencia, Duncan Edwards, and Helen McCain. 2016. Towards multispecies interaction environments: extending accessibility to canine users. In Proceedings of the Third International Conference on Animal-Computer Interaction. ACM, 8.

[44] Patricia J Manns and Karen E Chad. 2001. Components of quality of life for persons with a quadriplegic and paraplegic spinal cord injury. Qualitative health research 11, 6 (2001), 795-811.

[45] Roger W McIntire and Thomas A Colley. 1967. Social reinforcement in the dog. Psychological Reports 20, 3 (1967), 843-846.

[46] Michael J Muller. 2009. Participatory design: the third space in HCI. In Human-computer interaction. CRC press, 181-202.

[47] Kenichiro Noda. 2018. Google Home: smart speaker as environmental control unit. Disability and rehabilitation: assistive technology 13, 7 (2018), 674-675.

[48] Marguerite E O'Haire. 2013. Animal-assisted intervention for autism spectrum disorder: A systematic literature review. Journal of autism and developmental disorders 43, 7 (2013), 1606-1622.

[49] World Health Organisation. 2013. WHO factsheet on Spinal Cord Injury. (2013). https://www.who.int/ news-room/fact-sheets/detail/spinal-cord-injury

[50] Christina Papadimitriou. 2008. Becoming en-wheeled: the situated accomplishment of re-embodiment as a wheelchair user after spinal cord injury. Disability \& society 23, 7 (2008), 691-704.

[51] Kwang-Hyun Park, Zeungnam Bien, Ju-Jang Lee, Byung Kook Kim, Jong-Tae Lim, Jin-Oh Kim, Heyoung Lee, Dimitar H Stefanov, Dae-Jin Kim, Jin-Woo Jung, and others. 2007. Robotic smart house to assist people with movement disabilities. Autonomous Robots 22, 2 (2007), 183-198.

[52] Kwang-Hyun Park, Hyong-Euk Lee, Youngmin Kim, and Z Zenn Bien. 2008. A steward robot for human-friendly human-machine interaction in a smart house environment. IEEE Transactions on Automation Science and Engineering 5, 1 (2008), 21-25. 
[53] Emanuele Perini, Simone Soria, Andrea Prati, and Rita Cucchiara. 2006. FaceMouse: A human-computer interface for tetraplegic people. In European Conference on Computer Vision. Springer, 99-108.

[54] Petcube. 2020. PETCUBE BITES 2: Smart HD pet camera with treat dispenser, $180 \hat{A}$ r lens, and voice control. (2020). https://petcube. com/bites-2/

[55] PetSafe. 2020. Treat Train Remote Reward Dog Trainer. (2020).

https://store.intl.petsafe.net/en-gb/treat-and-train

[56] MW Post, AJ Dijk Van, FW Asbeck Van, and AJ

Schrijvers. 1998. Life satisfaction of persons with spinal cord injury compared to a population group.

Scandinavian Journal of Rehabilitation Medicine 30, 1 (1998), 23-30.

[57] Marti L Riemer-Reiss and Robbyn R Wacker. 2000. Factors associated with assistive technology discontinuance among individuals with disabilities. Journal of Rehabilitation 66, 3 (2000).

[58] Diana Rintala, Natalie Sachs-Ericsson, and K.A. Hart. 2002. The effects of service dogs on the lives of persons with mobility impairments: A pre-post study design. $S C I$ Psychosocial Process 15 (01 2002), 70-82.

[59] Charlotte Robinson, Clara Mancini, Janet van der Linden, Claire Guest, Lydia Swanson, Helen Marsden, Jose Valencia, and Brendan Aengenheister. 2015. Designing an emergency communication system for human and assistance dog partnerships. In Proceedings of the 2015 ACM International Joint Conference on Pervasive and Ubiquitous Computing. ACM, 337-347.

[60] Charlotte L Robinson, Clara Mancini, Janet Van Der Linden, Claire Guest, and Robert Harris. 2014. Canine-centered interface design: supporting the work of diabetes alert dogs. In Proceedings of the SIGCHI Conference on Human Factors in Computing Systems. ACM, 3757-3766.

[61] Kerri E Rodriguez, Jessica Bibbo, and Marguerite E O'Haire. 2018. The effects of service dogs on psychosocial health and wellbeing for individuals with physical disabilities or chronic conditions. Disability and rehabilitation (2018), 1-9.

[62] Nicola J Rooney, Steve Morant, and Claire Guest. 2013. Investigation into the value of trained glycaemia alert dogs to clients with type I diabetes. PloS one 8, 8 (2013), e69921.

[63] RSPCA. 2019. What is reward based dog training and why does the RSPCA support it? (Nov 2019). https:// kb.rspca.org.au/article-tags/reward-based-training/

[64] Carol Rushing. 1995. The effect of service dogs on the self-concept of spinal injured adults. Ph.D. Dissertation. ProQuest Information \& Learning.

[65] Natalie Sachs-Ericsson, Nancy K Hansen, and Shirley Fitzgerald. 2002. Benefits of assistance dogs: A review. Rehabilitation Psychology 47, 3 (2002), 251.
[66] Jason Seewoodhary, Thomas Dacruz, Erin Lloyd, and Peter J Evans. 2014. The role of diabetic alert dogs in the management of impaired hypoglycaemia awareness. Practical Diabetes 31, 8 (2014), 323-325.

[67] Vinod Sharma, Richard C Simpson, Edmund F LoPresti, Casimir Mostowy, Joseph Olson, Jeremy Puhlman, Steve Hayashi, Rory A Cooper, Ed Konarski, and Barry Kerley. 2008. Participatory design in the development of the wheelchair convoy system. Journal of NeuroEngineering and Rehabilitation 5, 1 (2008), 1.

[68] Emir Skejié and Marijan Durek. 2007. Assistive technologies for physically handicapped persons. In Innovations and Advanced Techniques in Computer and Information Sciences and Engineering. Springer, 173-178.

[69] Katharina Spiel, Christopher Frauenberger, Eva Hornecker, and Geraldine Fitzpatrick. 2017. When empathy is not enough: Assessing the experiences of autistic children with technologies. In Proceedings of the 2017 CHI Conference on Human Factors in Computing Systems. ACM, 2853-2864.

[70] SpinalCord.com. 2019. Quadriplegia: Tetraplegia. (2019).

https://www. spinalcord.com/quadriplegia-tetraplegia

[71] Clay Spinuzzi. 2005. The methodology of participatory design. Technical communication 52, 2 (2005), 163-174.

[72] Dimitar H Stefanov, Zeungnam Bien, and Won-Chul Bang. 2004. The smart house for older persons and persons with physical disabilities: structure, technology arrangements, and perspectives. IEEE transactions on neural systems and rehabilitation engineering 12, 2 (2004), 228-250.

[73] Molly Follette Story. 1998. Maximizing usability: the principles of universal design. Assistive technology 10, 1 (1998), 4-12.

[74] Philip Tedeschi, Aubrey H Fine, and Jana I Helgeson. 2010. Assistance animals: Their evolving role in psychiatric service applications. In Handbook on Animal-Assisted Therapy. Elsevier, 421-438.

[75] Dogs Trust. 2019. What is Positive Reinforcement Dog Training? (2019). https://www. dogstrustdogschool.org. uk/training/i-want-to-train-my-dog/ the-benefits-of-positive-reinforcement/

[76] Pet Tutor. 2020. Smart Training Feeder. (2020). https://smartanimaltraining.com/

[77] Dirk van der Linden and Anna Zamansky. 2017. Agile with animals: Towards a development method. In 2017 IEEE 25th International Requirements Engineering Conference Workshops (REW). IEEE, 423-426.

[78] Vappu Viemerö and Christina Krause. 1998. Quality of life in individuals with physical disabilities. Psychotherapy and psychosomatics 67, 6 (1998), 317-322. 
[79] Claude Vincent, Dany Gagnon, François Routhier, Jean Leblond, Pascale Boucher, Marie Blanchet, Valérie Martin-Lemoyne, and ADMI group. 2015. Service dogs in the province of Quebec: sociodemographic profile of users and the dogs' impact on functional ability.

Disability and rehabilitation: Assistive technology 10, 2 (2015), 132-140.

[80] Bess Williamson. 2019. Accessible America: A history of disability and design. Vol. 2. NYU Press.

[81] Chadwick A Wingrave, Jeremy Rose, Todd Langston, and Joseph J LaViola Jr. 2010. Early explorations of CAT: canine amusement and training. In CHI'10 Extended Abstracts on Human Factors in Computing Systems. ACM, 2661-2670.
[82] Melissa Winkle, Terry K Crowe, and Ingrid Hendrix. 2012. Service dogs and people with physical disabilities partnerships: A systematic review. Occupational therapy international 19, 1 (2012), 54-66.

[83] Mariko Yamamoto and Lynette A Hart. 2019. Professionally-and self-trained service dogs: Benefits and challenges for partners with disabilities. Frontiers in Veterinary Science 6 (2019).

[84] Gal Ziv. 2017. The effects of using aversive training methods in dogs-A review. Journal of veterinary behavior 19 (2017), 50-60. 